\title{
Preditores da Síndrome de Burnout em Trabalhadores da Saúde no Contexto Hospitalar
}

\author{
Janine Kieling Monteiro* \\ Universidade do Vale do Rio dos Sinos, Rio dos Sinos, Brasil \\ Mary Sandra Carlotto \\ Pontifícia Universidade Católica do Rio Grande do Sul, Porto Alegre, Brasil
}

\begin{abstract}
RESUMO
Esta pesquisa buscou identificar preditores sócios demográficos e laborais das dimensões de Burnout em 182 trabalhadores da saúde no contexto hospitalar. Foi utilizado um questionário, o Maslach Burnout Inventory e a Escala de Avaliação do Contexto do Trabalho. Na análise dos dados empregou-se a análise de regressão múltipla. Considerar a profissão estressante foi a variável de maior poder explicativo para as dimensões de Exaustão Emocional e baixa Realização Profissional. Como variáveis preditoras da Despersonalização destacaram-se condições negativas de trabalho, estresse no atendimento ao paciente e sexo masculino. O perfil de risco para Burnout foi constituído de profissionais com maior escolaridade; tempo de profissão; que percebem profissão, condições de trabalho, escala de trabalho, tipo e quantidade de pacientes como fatores estressantes.
\end{abstract}

Palavras-chave: pessoal de saúde; Burnout; hospital; trabalho.

\section{ABSTRACT \\ Predictors of Burnout Syndrome Among Healthcare Workers in Hospitals}

This research aims at identifying sociodemographic and laboral predictors of burnout syndrome in 182 health workers in the hospital setting. It uses a questionnaire, Maslach Burnout Inventory, and a working context scale. In the analysis of the data a multiple regression analysis was employed. Interviewees' considering their profession as stressful was the most revealing variable with regards to the Emotional Exhaustion dimension and the low level of Professional Efficacy. As predictor variables to Cynicism the research points to negative working environments, stress upon dealing with male patients. The risk profile for burnout syndrome includes higher schooled professionals, oldtimers and those who perceive their job, work scale, type and quantity of patients as stressing factors.

Keywords: Health personnel; burnout; hospital; work.

O trabalho em instituições hospitalares apresenta diversos estressores ocupacionais como ambiente insalubre, o regime de turnos, os plantões, os baixos salários, o contato muito próximo com os pacientes, mobilizando emoções e conflitos que tornam esses trabalhadores particularmente susceptíveis ao sofrimento psíquico e ao adoecimento devido ao trabalho (Rios, 2008). O profissional tem que manejar com pacientes em estado grave; compartilhar com o paciente e seus familiares sentimentos de angústia, a dor e o medo (Aranda-Beltrán et al., 2004) tendo que lidar inclusive com a possibilidade e/ou a morte de pacientes (Gerow, 2010). O exercício profissional é marcado por múltiplas exigências que se somam as condições desfavoráveis de trabalho (Avellar, Iglesias, \& Valverde, 2007) e as constantes reestruturações as quais o trabalhador deve se submeter e que lhe exigem constante adaptação (Nordang, Hall-Lord, \& Farup, 2010).

O trabalhador da saúde necessita lidar com a frustração dos inevitáveis fracassos, a depressão e o sentimento de impotência quando a realidade se impõe. Deve ser capaz de suportar esses sofrimentos inerentes à profissão e continuar desejando atuar num constante recomeçar (Rios, 2008). A interação entre profissional e clientela é, frequentemente, carregada de fortes emoções, como constrangimento, medo, frus- 
tração ou raiva de ambas as partes (Cherniss, 1980). A regularidade deste tipo de relação pode levar o profissional a vivenciar exaustão emocional, a adotar atitudes de distanciamento e sentir-se menos competente no seu trabalho, características que constituem a Síndrome de Burnout (SB) (Carlotto, 2009).

A SB, fundamentada na perspectiva sociopsicológica, constitui-se de três dimensões: exaustão emocional, despersonalização e baixa realização profissional. A exaustão emocional se caracteriza por uma falta ou carência de energia, entusiasmo e um sentimento de esgotamento de recursos. A despersonalização caracteriza-se por tratar os clientes, colegas e a organização de forma impessoal. É um estado psíquico em que prevalece o cinismo ou a dissimulação afetiva, a crítica exacerbada de tudo e de todos. Já a baixa realização profissional faz com que o trabalhador se autoavalie de forma negativa, sentindo-se infeliz e insatisfeito com o seu desenvolvimento profissional (Maslach \& Jackson, 1986).

A SB é uma doença multicausal, o seu desenvolvimento é influenciado por fatores individuais e laborais. Os fatores considerados estressores no trabalho são os que mais contribuem para desencadear a SB (Gil-Monte \& Peiró, 1997), tais como: sobrecarga de trabalho, condições de trabalho inadequadas (relacionadas às adoções de novas tecnologias e à estrutura organizacional), conflitos nas relações socioprofissionais e falta de perspectivas na carreira. Entre as variáveis individuais destacam-se: grande dedicação ao trabalho, tendência ao perfeccionismo, altas expectativas, indivíduos controladores e pessimistas, ter características relacionadas a dimensão Neuroticismo do modelo dos Cinco Grandes Fatores de Personalidade, entre outras (Maslach \& Leiter, 2008; World Health Organization, 1998).

A SB vem sendo investigada já há alguns anos em instituições hospitalares, devido ao crescente entendimento de que a saúde do trabalhador pode incidir na qualidade da prestação do serviço (Faller, Gates, Georges, \& Connelly, 2011; Pereda-Torales, Celedonio, Vásquez, \& Zamora, 2009). No Brasil, diversos estudos têm sido desenvolvidos com profissionais de saúde (Ebling, 2010; Jardim, Silva Filho, \& Ramos, 2004; Silva \& Carlotto, 2008; Soares \& Cunha, 2007; Tamayo, 2002) que identificam importantes associações entre burnout e as variáveis investigadas.
Burnout, geralmente, leva à deterioração do bemestar físico e mental. O profissional afetado se sente exausto, frequentemente está doente, sofre de insônia, úlcera, dores de cabeça, tensão muscular, fadiga crônica (Maslach \& Leiter, 1997) e depressão (Gil-Monte, 2009; Tomás-Sábado et al., 2010). No campo organizacional, a SB está altamente associada à insatisfação dos trabalhadores, absenteísmo, rotatividade de pessoal (Knight \& Leimer, 2010; Swider \& Zimmerman, 2010) e baixos níveis de comprometimento no trabalho (Demerouti, Mostert, \& Bakker, 2010). Um estudo de revisão da literatura sobre Burnout (Trigo, Teng, \& Hallak, 2007) em base de dados nacionais e internacionais, entre os anos de 1985 e 2006, indicou que a síndrome se apresenta frequentemente associada à depressão e comorbidades. Além disto, os autores destacam que os efeitos negativos da SB abrangem três níveis: individual (que traz prejuízos físico, mental, profissional e social), profissional (atendimento descuidado e lento ao cliente, contato impessoal com colegas de trabalho e/ou pacientes/clientes) e organizacional (conflitos com a equipe, absenteísmo, diminuição da qualidade e quantidade dos serviços).

Segundo Rosa e Carlotto (2006), verifica-se uma tendência das organizações hospitalares no investimento da estrutura física, mais especificamente, estéticas de suas instalações, com o intuito de gerar avaliação positiva no usuário, estando essa questão relacionada ao mercado consumidor. No entanto, os profissionais que trabalham na instituição precisam, acima de tudo, de melhores condições e organização de trabalho, com suporte de seus supervisores, benefícios e políticas organizacionais que contemplem sua qualidade de vida no trabalho.

Estudo realizado em um hospital com profissionais da área da saúde realizado por Borges, Argolo e Baker (2006) encontrou que quanto menor a renda do profissional, maior era a tendência a apresentar comportamentos de indiferença e ceticismo na relação com o usuário e colegas de trabalho. Investigação desenvolvida por Rosa e Carlotto (2006) identificou associação negativa entre as dimensões de exaustão emocional e despersonalização e a idade dos trabalhadores. Com relação aos fatores de estresse, Silva e Carlotto (2008) verificaram que quanto maior a percepção de que a profissão é estressante assim como a carga horária, a escala, o tipo de paciente atendido, as condições de 
trabalho e a dificuldade em conciliar trabalho e família, maior é o sentimento de exaustão emocional nos profissionais de saúde. A despersonalização aumentava com a ocorrência do sentimento de insatisfação com as relações hierárquicas, com o menor tempo de experiência profissional e com o número de dias de licença-saúde. Já a realização profissional elevava-se na medida em que aumenta a satisfação com as relações hierárquicas, com o ambiente físico de trabalho, com o conteúdo das atividades desenvolvidas e com as oportunidades de crescimento profissional. Quanto maior a carga horária no trabalho, maior era a realização profissional. Já, perceber como estressante conciliar família e trabalho diminui o sentimento de que o trabalho é elemento de realização profissional. Achkar (2006), em estudo realizado com médicos e enfermeiras, identificou relação inversa entre Burnout e qualidade de vida no trabalho e diferenças nos níveis entre os dois grupos, tendo os médicos apresentado maiores níveis. Sandoval, González, Sanhueza, Borjas e Odgers (2005) encontraram 22,72\% de Burnout em profissionais de um hospital chileno. Sua presença foi predominantemente em mulheres, trabalhadores solteiros, em profissionais na faixa de 24 a 40 anos e com experiência profissional inferior a 10 anos. Ebling (2010) em estudo realizado com profissionais da saúde de um hospital público identificou um perfil de risco constituído por homens, trabalhadores jovens, em processo de formação em nível de graduação, com menor remuneração, maior número de pacientes atendidos por dia, médicos, profissionais concursados, insatisfeitos com o trabalho, com desejo de abandonar a profissão, a instituição e a função na instituição.

Um estudo recente de revisão de literatura procurou investigar a presença da SB em profissionais de saúde (Ferrari, França \& Magalhães, 2012) em artigos de língua portuguesa, foram analisados 16 artigos referentes ao período entre outubro e dezembro de 2010, os autores apontaram que a revisão evidenciou forte associação entre a SB e os profissionais de saúde. Estes profissionais são propensos ao elevado nível de estresse pelo fato de terem ocupações assistenciais onde a prestação de cuidados ao próximo, baseada em fundamentos humanísticos, torna-se prioridade. $\mathrm{Ne}$ nhum dos estudos avaliados apresentou um índice de prevalência de SB preciso na amostra estudada, provavelmente porque a maioria utilizou o MBI como instrumento, sendo que este ainda não possui dados de validação para uso no Brasil (Carlotto \& Câmara, 2008). Além disto, nenhum destes estudos buscou analisar fatores preditivos da SB entre os profissionais de saúde.

No entanto, algumas pesquisas apontam fatores preditivos da SB, tais como: ter menor suporte da chefia (Souza \& Silva, 2002); ter avaliação mais negativa e ver incongruências relacionadas a questões éticas (justiça e valores organizacionais) no local de trabalho (Maslach \& Leiter, 2008). A ausência de coleguismo, a sobrecarga de trabalho e o conflito nos valores e práticas organizacionais revelaram-se preditores para a dimensão de Exaustão Emocional (Tamayo, 2009).

A maioria das discussões sobre Burnout pontua que a síndrome é resultante de fatores pessoais e ambientais, contudo, estudos apontam que as características do trabalho são mais relacionadas ao Burnout (Maslach, Schaufeli \& Leiter, 2001). Assim, o presente estudo observacional analítico de corte transversal (Grimes \& Schulz, 2002) tem como objetivo identificar os preditores sociodemográficos e laborais das dimensões de Burnout em profissionais da saúde que atuam no contexto hospitalar.

\section{MÉTODO}

\section{Participantes}

Fizeram parte do estudo 182 trabalhadores da saúde que atuam em dois hospitais, sendo que 92 sujeitos desenvolvem as suas atividades em um hospital público e 90 sujeitos em um hospital da rede privada. O grupo de participantes era composto por 66 técnicos de enfermagem, 55 enfermeiros e 61 médicos. A maior parte da população em estudo é constituída por mulheres $(68,7 \%)$, casados $(54,9 \%)$, com nível superior $(68,7 \%)$, que trabalham em mais de um local $(51,1 \%)$ e tem uma jornada de trabalho correspondente a 30 horas semanais $(54,9 \%)$.

No que diz respeito à descrição das variáveis intervalares, a idade dos participantes variou de 18 a 65 anos, com idade média de 36,48 anos e desvio padrão de 10,01; o tempo de profissão de um a 40 anos, com média de 11,41anos e desvio padrão de 8,27 e o tempo no local foi de um a 30 anos, com média de 8,5 anos e desvio padrão de 7,44 . 


\section{Instrumentos}

Para o levantamento de variáveis sócio demográficas (sexo, idade, estado civil, escolaridade, problemas de saúde) e laborais (tempo de profissão, tempo no local de trabalho, número de pacientes, carga horária, realização de horas extras, trabalhar em outro loca, modelo da profissão, afastamento do trabalho, ter gozado de férias; pensar em desistir da profissão) foi elaborado um questionário autoaplicado. Este instrumento foi desenvolvido especificamente para responder aos objetivos do estudo, tendo como base principal o referencial teórico sobre a Síndrome de Burnout em profissionais da área da saúde. Além disto, também foi elaborada uma escala para avaliar a percepção de fatores estressores relacionados ao seu trabalho, com seis itens, a qual possuía uma escala de pontuação variando de 1 a 5 (1=nada estressante, $2=u m$ pouco estressante, $3=$ indiferente, $4=$ bastante estressante e $5=$ muito estressante). Nesta avaliação era solicitado que o participante circulasse o número que ele considerava expressar a sua opinião. Por exemplo, você considera a sua profissão ( $1=$ nada estressante, $2=u m$ pouco estressante, $3=$ indiferente, $4=$ bastante estressante e $5=$ =muito estressante). Outros fatores investigados foram a carga horária, a escala de trabalho, o tipo de paciente atendido, as condições de trabalho e conciliar família e profissão.

Para avaliar a Síndrome de Burnout foi utilizado o MBI - Maslach Burnout Inventory - HSS (Maslach \& Jackson, 1986) que tem a tradução validada para a língua portuguesa por Lautert (1997). O inventário é auto-aplicado e avalia como o sujeito vivencia seu trabalho, de acordo com as três dimensões propostas pelo modelo teórico de Maslach: Exaustão Emocional (EE) (9 itens), Realização Pessoal no Trabalho (RP) (8 itens) e Despersonalização (DE) (5 itens). Totaliza, portanto, 22 itens que indicam a frequêencia das respostas com uma escala de pontuação variando de $1 \mathrm{a}$ 7. Neste estudo, adotou-se o sistema de pontuação de 1 a 5, utilizado por Tamayo (1997) na adaptação brasileira do instrumento. Utilizou-se, portanto, 1 para "nunca", 2 para "raramente", 3 para "algumas vezes", 4 para indicar "frequentemente" e 5 para "sempre". A consistência interna das três dimensões do inventário é satisfatória, pois apresenta um alfa de Cronbach que vai de 0,71 até 0,90 (Maslach \& Jackson, 1986).
Outro instrumento usado na pesquisa foi a Escala de Avaliação do Contexto do Trabalho (EACT), desenvolvida por Mendes e Ferreira (2007), a qual foi utilizada para avaliar algumas variáveis laborais. De forma mais específica, este instrumento analisa três fatores: organização do trabalho (11 questões com confiabilidade de 0,72 que se referem à liberdade, ritmo e carga de trabalho), relações socioprofissionais (10 itens com confiabilidade de 0,87 que investigam aspectos diversos da relação entre colegas e chefias) e condições de trabalho (10 itens de confiabilidade 0,89 , que avaliam aspectos físicos e materiais). A escala é do tipo likert composta de cinco pontos que vão de 1 a 5 (1=nunca, $2=$ raramente, $3=$ às vezes, $4=$ frequentemente, $5=$ sempre).

\section{Procedimentos}

Primeiramente, buscou-se a apreciação e autorização do projeto pela direção dos Hospitais, e a devida comunicação às chefias e gerências dos setores. Assim, após esta etapa, foi selecionada a amostra e os sujeitos foram contatados pelos pesquisadores, que explicaram o objetivo da investigação, forneceram informações quanto ao preenchimento dos questionários e sobre as questões éticas envolvidas. A coleta dos dados foi realizada entre os meses de novembro de 2008 a março de 2009.

A pesquisa obteve a aprovação do Comitê de Ética da Universidade, e observou os procedimentos éticos conforme Resolução 196 do Conselho Nacional de Saúde (CNS), no que diz respeito à pesquisa com seres humanos (Ministério da Saúde do Brasil, 1996).

O banco de dados foi digitado e, posteriormente, analisado através do SPSS (Statistical Package for the Social Sciences) - versão 18. Após realizaram-se análises descritivas de caráter exploratório a fim de avaliar a distribuição dos itens, casos omissos e identificação de extremos, foram verificados os pressupostos de multicolinearidade, singularidade e normalidade para o desenvolvimento de análises de regressão. Como critérios para as análises de regressão, foi verificada a distribuição normal dos resíduos (através do Teste Kolmogorov-Smirnov), também, foi utilizado o teste de Durbin-Watson para considerar o diagnóstico de independência dos resíduos, assim, foram obtidos índices satisfatórios em todas as análises rodadas. Em relação a adequação do tamanho da amostra ao número de variáveis incluídas no modelo, segundo Hair, 
Black, Babin, Anderson e Tatham (2009) uma regressão múltipla requer uma amostra mínima de 100 observações para a maioria das situações de pesquisa, portanto uma amostra de 182 participantes pode ser considerada satisfatória para as análises realizadas neste estudo.

Posteriormente, foi realizada análise de regressão linear múltipla para identificar os preditores de cada dimensão em cada grupo, adotando-se nível de significância de 5\%. Foram realizadas três análises de regressão múltipla pelo método stepwise, tendo cada uma como variável dependente a dimensão do MBI correspondente, ou seja: exaustão emocional, despersonalização e realização profissional, e como variáveis independentes, o conjunto de variáveis sócio demográficas (sexo, idade, estado civil, escolaridade, número de filhos, problemas de saúde no último ano) e laborais (modelo da profissão; tempo de experiência na profissão; tempo de empresa; carga horária; núme- ro de pacientes atendidos; realização de horas extras; trabalhar em outro local; afastamento no trabalho; avaliação da organização do trabalho, relações socioprofissionais e condições de trabalho; avaliação do estresse relacionado a profissão, a carga horária, a escala de trabalho, o tipo de paciente atendido, as condições de trabalho e conciliar família e profissão).

\section{RESULTADOS}

Resultados obtidos na dimensão de exaustão emocional apontam para um modelo preditor composto pelas variáveis: estresse/profissão, pensar em desistir da profissão, estresse/condições de trabalho, escolaridade, número de pacientes, estresse/escala de trabalho e organização do trabalho (Tabela 1). Estes fatores, conjuntamente, explicam $64 \%$ da variabilidade da exaustão emocional. A variável com maior poder explicativo refere-se à percepção de que sua profissão é estressante com $36,1 \%$ de variância.

\section{Tabela 1}

Variáveis Preditoras das Dimensões da Síndrome de Burnout

\begin{tabular}{|c|c|c|c|c|c|}
\hline Variáveis & $\mathbf{R}$ & $\mathbf{R}^{2}$ ajustado & Beta & $\mathbf{t}$ & $p$ \\
\hline \multicolumn{6}{|l|}{ Exaustão Emocional } \\
\hline 1. Estresse/profissão & 0,601 & 0,361 & 0,249 & 3,843 & 0,000 \\
\hline 2. Desistir da profissão & 0,698 & 0,487 & 0,291 & 5,305 & 0,000 \\
\hline 3. Estresse/condições de trabalho & 0,729 & 0,532 & 0,179 & 2,669 & 0,008 \\
\hline 4. Escolaridade & 0,750 & 0,563 & 0,210 & 3,720 & 0,000 \\
\hline 5. Número de pacientes & 0,764 & 0,584 & 0,196 & $-3,844$ & 0,000 \\
\hline 6. Estresse/escala de trabalho & 0,776 & 0,602 & 0,159 & 2,185 & 0,030 \\
\hline 7. Organização do trabalho & 0,786 & 0,618 & 0,144 & 2,621 & 0,010 \\
\hline 8. Tempo de profissão & 0,794 & 0,630 & 0,249 & 2,436 & 0,016 \\
\hline $\begin{array}{l}\text { 9. Estresse/conciliar trabalho e família } \\
\text { Valor do Durbin-Watson=2,04 }\end{array}$ & 0,800 & 0,640 & 0,291 & 1,978 & 0,050 \\
\hline \multicolumn{6}{|l|}{ Despersonalização } \\
\hline 1. Condições de trabalho & 0,337 & 0,114 & 0,267 & 3,501 & 0,001 \\
\hline 2. Estresse/paciente & 0,389 & 0,152 & 0,193 & 2,531 & 0,012 \\
\hline $\begin{array}{l}\text { 3. Sexo } \\
\text { Valor do Durbin-Watson }=2,24\end{array}$ & 0,415 & 0,172 & 0,146 & 1,988 & 0,049 \\
\hline \multicolumn{6}{|l|}{ Realização Profissional } \\
\hline 1. Estresse/profissão & 0,394 & 0,155 & $-0,334$ & 34,826 & 0,000 \\
\hline 2. Relações socioprofissionais & 0,442 & 0,195 & $-0,202$ & $-4,621$ & 0,000 \\
\hline 3. Sexo & 0,464 & 0,215 & $-0,186$ & $-2,796$ & 0,006 \\
\hline 4. Tempo no local de trabalho & 0,491 & 0,241 & 0,186 & $-2,618$ & 0,010 \\
\hline $\begin{array}{l}\text { 5. Afastamento do trabalho } \\
\text { Valor do Durbin-Watson=1,95 }\end{array}$ & 0,517 & 0,267 & $-0,163$ & 2,604 & 0,010 \\
\hline
\end{tabular}


Com relação à despersonalização, o modelo final explicou $17,2 \%$ da variabilidade, sendo detectadas como preditoras as variáveis condições de trabalho, considerar o atendimento ao paciente um fator de estresse e sexo (Tabela 1). A variável com maior poder explicativo $(11,4 \%)$ foi a que avaliou as condições de trabalho.

Por fim, a análise de regressão múltipla para a dimensão de realização profissional, revelou como preditoras as variáveis: estresse/profissão, relações de trabalho, sexo, tempo no local de trabalho, afastamento do trabalho e a percepção de que a profissão é estressante com $15,5 \%$, tendo o modelo final explicado $26,7 \%$ da variabilidade total desta dimensão (Tabela 1 ).

Os resultados evidenciam relação positiva, com exceção do número de pacientes atendidos, com todas as variáveis do modelo explicativo revelando aumentar a exaustão emocional. As variáveis relacionadas à despersonalização também indicam associação positiva. Já as variáveis que explicam a realização profissional, com exceção do tempo de trabalho, relevam associação negativa.

\section{DISCUSSÃO}

A análise de preditores indica predominância das variáveis laborais e psicossociais nas três dimensões da Síndrome de Burnout. Este resultado parece confirmar outros estudos que apontam serem as variáveis laborais as mais relacionadas ao Burnout (Gil-Monte, 2009; Boada, Vallejo, \& Agullo-Thomás, 2004).

A dimensão de exaustão emocional apresentou um modelo preditor composto pelas variáveis perceber sua profissão como estressante, ter mais tempo de profissão, perceber as condições de trabalho como importante fator de estresse assim como a escala de trabalho, a sua organização e a elevada quantidade de pacientes que o trabalhador necessita atender. Verifica-se que estas variáveis explicativas se referem a aspectos relacionados às condições e à organização do trabalho, geralmente com implicações que envolvem ambiente físico e materiais mais precários e uma sobrecarga intensa. A sobrecarga laboral é um preditor da exaustão emocional (Maslach \& Leiter, 1997). O atendimento ao paciente implica não somente em ter um horário dedicado ao mesmo, mas também as diversas exigências burocráticas como preenchimento de formulários, realização de prescrições, realização de relatórios, dentre outras (Kareaga, Exeberria, \& Smith, 2009). Também explicam esta dimensão a elevada escolaridade dos profissionais e o pensamento frequente em pensar em desistir da profissão. Profissionais com maior escolaridade, por terem realizado maior investimento profissional, podem desenvolver maiores expectativas com seu contexto e atividade profissional. Além disto, eles têm maior responsabilidade técnica do que os profissionais que fazem as tarefas mais operacionais, como os técnicos de enfermagem. Segundo Cordes e Dougherty (1993), altas discrepâncias entre as expectativas de desenvolvimento profissional e aspectos reais de seu trabalho propiciam a ocorrência de maiores níveis de Burnout. Estas questões, sem dúvida, ocasionam um maior questionamento sobre a possibilidade de mudar de profissão. Já com relação à percepção de que conciliar trabalho e família é uma questão estressante, tem-se encontrado certo consenso na literatura quanto ao seu risco potencial de adoecimento nas profissões assistenciais (Ayo, Henry, \& Adebukola, 2009; Cinamon \& Rich, 2010).

É importante destacar que a variável com maior poder explicativo se refere à percepção de que sua profissão é estressante. Este resultado corrobora o encontrado em outros estudos (Mallmann, Palazzo, Carlotto, \& Aerts, 2009; Silva \& Carlotto, 2008). Brock e Grady (2000) referem que profissionais que reconhecem os estressores da sua profissão mobilizam estratégias de enfrentamento para lidar com os mesmos, podendo esta percepção de maior conscientização refletir em uma elevação nos níveis de exaustão emocional.

A despersonalização é uma resposta negativa que produz sentimento de difícil controle e que interfere no desempenho profissional. A variável de maior poder explicativo foi a avaliação negativa das condições de trabalho seguida pela de considerar o atendimento ao paciente um fator de estresse, sendo o sexo masculino o mais propenso a desenvolver distanciamento em relação à clientela atendida. Este resultado tem sido consolidado na literatura que aponta ser esta dimensão mais frequente em trabalhadores do sexo masculino (Nordang et al., 2010; Vercambre, Brosselin, Gilbert, Nerrière, \& Kovess-Masféty, 2009), devido ao fato dos homens apresentarem maior dificuldade para lidar com os estressores interpessoais (Kulik, 2006) e com tarefas relacionadas ao cuidado da saúde, 
culturalmente vistas como tarefas femininas. Este resultado tem sido explicado pelos aspectos culturais decorrente do processo de socialização que não permite ao homem uma maior expressão de sentimentos (Aryee, 1993; Ushiro \& Nakayama, 2010).

$\mathrm{Na}$ análise da realização profissional, o modelo explicativo é constituído pelas variáveis: percepção de que a profissão é estressante, inadequadas relações socioprofissionais, sexo feminino, maior tempo de trabalho na instituição, ter sido afastada do trabalho por problemas de saúde. A maior realização profissional em mulheres pode estar relacionada às características da emocionalidade das profissões assistenciais como também pela questão de que expectativas de sucesso, competição e desenvolvimento (Schaufeli \& Greenglass, 2001). Trabalhar a mais tempo no local aumenta a realização, provavelmente pelo fato do profissional com mais tempo de trabalho possuir maior segurança na realização de tarefas e ter desenvolvido relações de trabalho mais significativas, o que também explicaria a menor realização quando existem relações interpessoais danosas.

Verifica-se que considerar a profissão estressante foi a variável de maior pode explicativo para a dimensão de desgaste emocional e a baixa realização profissional, indo ao encontro do Modelo de Burnout de Gil-Monte e Peiró (1997), o qual postula que a SB se inicia com o sentimento de baixa realização pessoal e esgotamento emocional em paralelo. A despersonalização seria desencadeada como estratégia defensiva ou de enfrentamento. Neste sentido, pode-se pensar na possibilidade de um processo inicial da síndrome por meio da avaliação negativa sobre a profissão exercida.

Todavia, ressalta-se que se deve ter cautela com relação aos resultados obtidos, uma vez que estes são decorrentes de hospitais localizados em uma região específica do sul do Brasil, não sendo, portanto, passíveis de generalizações. É importante considerar, ainda, o efeito do trabalhador sadio, questão peculiar em estudos transversais em epidemiologia ocupacional que, muitas vezes, exclui o possível doente (Mc Michael, 1976). Essa é uma situação que pode subestimar o tamanho dos riscos identificados, porque os mais afetados não conseguem manter-se no emprego, afastando-se geralmente por licenças para tratamento de saúde. Também se deve considerar a participação voluntária, podendo os com maior nível de burnout ter optado por não participar do estudo (Pas, Bradshaw, Hershfeldt, \& Leaf, 2010).

A investigação alude à necessidade de aprofundamento dos resultados obtidos, assim, sugere-se a realização de novos estudos, com inclusão de outras variáveis e delineamentos em contextos hospitalares diferenciados. Outra possibilidade de pesquisa seria estudar os profissionais da saúde que tiveram que ser afastados do trabalho.

Identificar o perfil de risco e os sinais precoces de desenvolvimento do Burnout é de fundamental importância para intervenções preventivas (Maslach \& Leiter, 2008). O estudo aponta para um perfil de risco constituído de profissionais de ambos os sexos, com maior escolaridade, tempo de profissão, menos tempo no local de trabalho, que possuem a percepção de que a profissão é estressante, que consideram estressantes as condições de trabalho, a escala de trabalho, o tipo e a quantidade de pacientes que atendem e a dificuldade em conciliar trabalho e família. Organização e condições de trabalho consideradas inadequadas, juntamente com as relações sócio profissionais mais conflituosas e já ter tido afastamento no trabalho finalizam o perfil encontrado. A maioria dos resultados encontrados aponta para questões de ordem contextual e laboral. De acordo com Carlotto (2010), frente às psicopatologias laborais e o sofrimento psíquico no trabalho, mudanças profundas necessariamente envolvem perspectivas políticas, sociais e organizacionais, ou seja, um modelo interventivo de prevenção à Burnout abarcaria um conjunto complexo de ações, que abrangesse fundamentalmente a escuta e participação dos trabalhadores envolvidos.

\section{REFERÊNCIAS}

Achkar, T. C. S. (2006). Síndrome de Burnout: repercussões na qualidade de vida no trabalho de profissionais de saúde de um hospital privado da cidade de Cascavel$P R$. Dissertação de mestrado não publicada, Programa de Pós-Graduação em Psicologia, Universidade Católica Dom Bosco.

Aranda-Beltrán, C., Pando-Moreno, C. M., Salazar-Estrada, J. G., Torres-López, T. M., Aldrete-Rodríguez, M. G., \& Pérez-Reyes, M. B. (2004). Factores psicosociales laborales y Síndrome de Burnout en médicos del primer nivel de atención. Disponível em: <www.cucs.udg.mx/invsa lud/abril2004/art4.html.> Acesso em: 12 dez. 2004. 
Aryee, S. (1993). Dual-earner couples in Singapure: an examination of work and nonwork sources of their experienced burnout. Human Relations, 46(12), 1441-1469.

Avellar, L.Z., Iglesias, A., \& Valverde, P. V. (2007). Sofrimento psíquico em trabalhadores de enfermagem de uma unidade de oncologia. Psicologia em Estudo, 12(3), 475-481.

Ayo, H. T., Henry, S., \& Adebukola, K. T. (2009). Psychosocial variables as predictors of work-family conflict among secondary school teachers in irele local government area, Ondo State, Nigeria. Pakistan Journal of Social Sciences, 6(1), 11-18.

Boada, J., Vallejo, R. D., \& Agulló-Tomás, E. (2004). El Burnout las manifestaciones psicosomáticas como consecuentes del clima organizacional y de la motivación laboral. Psicothema, 16, 125-131.

Borges, L. O., Argolo, J. C. T., \& Baker, M. C. S. (2006). Os valores organizacionais e a Síndrome de Burnout: dois momentos em uma maternidade pública. Psicologia: Reflexão e Crítica, 19(1), 34-43.

Brasil. Ministério da Saúde. (1996). Diretrizes e Normas Regulamentadoras de Pesquisa Envolvendo Seres Humanos. Resolução 196/96 do Conselho Nacional de Saúde.

Brock, B. L., \& Grady, M. L. (2000). Rekindling the flame: principals combating teacher burnout. Thousand Oaks: Corwin Press.

Carlotto, M. S. (2009). A relação profissional-paciente e a Síndrome de Burnout. Encontro: Revista de Psicologia, 12(17), 7-20.

Carlotto, M. S. \& Câmara, S. G. (2008). Análise de Produção Científica de Burnout no Brasil. Revista Psico, 39(2), 152-158.

Cherniss, C. (1980). Professional burnout in human service organizations. New York: Praeger

Cinamon, R. G., \& Rich, Y. (2010). Work Family Relations: antecedents and outcomes. Journal of Career Assessment, 18(1) 59-70.

Cordes C. L., \& Dougherty, T. W. (1993). A review and integration of research on job burnout. Academy of $\mathrm{Ma}$ nagement Review, 18(4), 632- 636.1

Demerouti, E. Mostert, K., \& Bakker, A. B. (2010). Burnout and work engagement: a thorough investigation of the independency of both constructs. Journal of Occupational Health Psychology, 15(3), 209-222.

Draper, N. R.; Smith, H. (1981). Applied Regression Analysis. JohnWiley \& Sons: New York.

Ebling, M. (2010). Síndrome de burnout em profissionais da saúde de um hospital público do Paraná. Dissertação de Mestrado. Mestrado em Saúde Coletiva. Universidade Luterana do Brasil, Canoas, Brasil.

Faller, M. S., Gates, M. G., Georges, J. M., \& Connelly, C. D. (2011). Work-related burnout, job satisfaction, intent to leave, and nurse-assessed quality of care among travel nurses. Journal of Nursing Administration, 41(2), 71-77.

Ferrari, R., França F. M. de, \& Magalhães, J. (2012). Avaliação da síndrome de burnout em profissionais de saúde: uma Revisão integrativa da literatura. Revista Eletrônica Gestão \& Saúde, 3(3), 1150-165.

Gerow, L. et al. (2010). A phenomenological study of nurses'experience of grief following patient death. Western Journal of Nursing Research, 31(8) 1078-1079.

Gil-Monte, P. R. (2009). Algunas razones para considerar los riesgos psicosociales en el trabajo y sus consecuencias en la salud pública. Revista Española de Salud Pública, 83(2),169-173.

Gil-Monte, P. R., \& Peiró, J. M. (1997). Desgaste psíquico en el trabajo: el síndrome de quemarse. Madrid: Síntesis.

Grimes, D. A., \& Shulz, K. F. (2002). An overview of clinical research: the lay of the land. The Lancet, 359, 57-61.

Hair, J. F.; Black, W. C.; Babin, B. J.; Anderson, R. E. \& Tatham, R. L. (2009). Análise Multivariada de Dados. 6ed. Porto Alegre: Bookman.

Jardim, S. R., Silva Filho, J., \& Ramos, A. (2004). O diagnóstico de Burnout na atenção em saúde mental dos trabalhadores. Em A. Araújo et al. (Orgs.). Cenários do trabalho: subjetividade, movimento e enigma. (pp. 7387). Rio de Janeiro: DP \& A.

Kareaga, A. A., Exeberria, S. A., \& Jonathan C. Smith (2009). Assessment of burnout and psychological wellbeing among health professionals in the Basque Country. Psychology in Spain, 13(1), 62-71.

Knight, W. E., \& Leimer, C. L. (2010). Will IR staff stick? An exploration of institutional researchers' intention to remain in or leave their jobs. Research in Higher Education, 51(2), 109-131.

Kulik, L. (2006). Burnout among volunteers in the social services: the impact of gender and employment status. Journal of Community Psychology, 34(5), 541-561.

Lautert, L. (1997). O desgaste profissional: uma revisão da literatura e implicações para a enfermeira. Revista Gaúcha Enfermagem, 18(2), 83-93.

Mallmann, C. S., Palazzo, L. S., Carlotto, M. S., \& Aerts, D. R. G. de C. (2009). Fatores associados à síndrome de burnout em funcionários públicos municipais. Psicologia: Teoria e Prática, 11(2), 69-82.

Maslach, C., \& Jackson, S. E. (1981). The measurement of experienced burnout. Journal of Occupational Behavior, 2, 99-113.

Maslach, C., \& Jackson, S. E. (1986). Maslach Burnout Inventory. $2^{\mathrm{a}}$.ed. Palo Alto, CA: Consulting Psychologist Press.

Maslach, C., \& Leiter, M. P. (1997). The truth about burnout: how organization cause, personal stress and what to do about It. San Francisco: Jossey-Bass. 
Maslach, C., \& Leiter, M. P. (2008). Early predictors of job burnout and engagement. Journal of Applied Psychology, 93(3), 498-512.

Maslach, C., Schaufeli, W. B., \& Leiter, M. P. (2001). Job burnout. Annual Review Psychology, 52, 397-422.

Mc Michael, A. J. (1976). Standardized mortality ratios and the healthy worker effect: scratching beneath the surface. Journal Occupational Medical, 18, 165-168.

Mendes, A. M., \& Ferreira, M. C. (2007). Inventário sobre trabalho e riscos de adoecimento - ITRA: Instrumento auxiliar de diagnóstico de indicadores críticos no trabalho. Em A M. Mendes (Org.). Psicodinâmica do trabalho: teoria, método e pesquisas (pp. 111-126). São Paulo: Casa do Psicólogo.

Nordang, K., Hall-Lord, M-L., \& Farup, P. G. (2010). Burnout in health-care professionals during reorganizations and downsizing. A cohort study in nurses. BMC Nursing, 9,8. Disponível em: <http://www.biomedcen tral.com/1472-6955/9/8>. Acesso em: 12 dez. 2004.

Pas, E. T. Bradshaw, C. P., Hershfeldt, P. A., \& Leaf, P. J. (2010). A multilevel exploration of the influence of teacher efficacy and burnout on response to student problem behavior and school-based service use. School Psychology Quarterly, 25(1), 13-27.

Pereda-Torales, L., Celedonio, F. G. M., Vásquez, M. T. H., \& Zamora, M. I. Y. (2009). Síndrome de burnout en médicos y personal paramédico. Salud Mental, 32, 399404.

Rios, I. C. (2008). Humanização e ambiente de trabalho na visão de profissionais da saúde. Saúde e Sociedade, 17(4), 151-160.

Rosa, C. da, \& Carlotto, M. S. (2006). Síndrome de Burnout e satisfação no trabalho em_profissionais de uma instituição hospitalar. Revista da SBPH, 8(2), 1-15.

Sandoval, C., González, P., Sanhueza, L., Borjas, R., \& Odgers, C. (2005). Síndrome de Burnout en el personal profesional del hospital de Yumbel. Apresentado em Congreso Nacional, 12, Congreso Científico Gremial, 7, Santiago do Chile. pp.1-10.

Schaufeli, W. B. \& Greenglass, E. R. (2001). Introduction to special issue on burnout and health. Psychology and Health, 16, 501-510.

Schulz, R., Greenley, J. R., \& Brown, R. (1995). Organization, management, and client effects on staff burnout. Journal of Health and Social Behavior, 36(4), 333-353.

Silva, T. D. da, \& Carlotto, M. S. (2008). Síndrome de Burnout em trabalhadores da enfermagem de um hospital geral. Revista da SBPH, 11(1), 113-130.

Soares, H. L. R., \& Cunha, C. E. C. (2007). A síndrome do "burn-out": sofrimento psíquico nos profissionais de saúde. Revista do Departamento de Psicologia da UFF, 19(2), 505-506.
Souza W.C, Silva A.M.M. (2002). A influência de fatores de personalidade e de organização do trabalho no burnout em profissionais de saúde. Estudos de Psicologia, 19 (1): 37-48.

Squires, A \& Livesley, B. (1984). Beware of burnout. Journal of the Chartered Society of Physiotherapy, 70, 235-238.

Swider, B., \& Zimmerman, R. (2010). Born to burnout: a meta-analytic path model of personality, job burnout, and work outcomes. Journal of Vocational Behavior, 76(3), 487-506.

Tamayo, M. R. (2009). Burnout: implicações das fontes organizacionais de desajuste indivíduo-trabalho em profissionais de enfermagem. Psicologia: Reflexão e Crítica, Porto Alegre, 22(3), 474-482.

Tamayo, M. R. (2002). Burnout: relação com a afetividade negativa, o coping no trabalho e a percepção de suporte organizacional. Tese de Doutorado não publicada, Instituto de Psicologia, Universidade de Brasília, Brasília.

Tamayo, M. R. (1997). Relação entre a síndrome do burnout e os valores organizacionais no pessoal de enfermagem de dois hospitais públicos. Dissertação de mestrado não-publicada, Universidade de Brasília, Brasília.

Trigo, T. R.; Teng, C. T. \& Hallak, J. E. C. (2007). Síndrome de burnout ou estafa profissional e os transtornos psiquiátricos. Revista Psiquiátrica Clínica, 34(5), 223233.

Tomás-Sábado, J., Maynegre-Santaulària, M, Pérez-Bartolomé, M, Alsina-Rodríguez, M, Quinta-Barbero, R., \& Granell-Navas, S. (2010). Burnout Syndrome and Suicide Risk Among Primary Care Nurses. Enfermagem Clínica, 20(3), 173-178.

World Health Organization. (1998). Guidelines for the primary prevention of mental, neurological and psychosocial disorders: Staff Burnout. In: Geneva Division of Mental Health World Health Organization, pp. 91-110.

Ushiro, R., \& Nakayama, K. (2010). Gender role attitudes of hospital nurses in Japan: Their relation to burnout, perceptions of physician-nurse collaboration, evaluation of care, and intent to continue working. Journal of Nursing Science, 7, 55-64.

Vercambre, M. N., Brosselin, P., Gilbert, F., Nerrière, E., \& Kovess-Masféty, V. (2009). Individual and contextual covariates of burnout: a cross-sectional nationwide study of French teachers. BMC Public Health,10(9),333. Disponível em: <http://www.biomedcentral.com/1471-2458/ 9/333>. Acesso em: 18 out. 2007.
Recebido em: 09/07/2012

Última revisão em: 05/07/2013 Aceito em: 26/11/2013 